Binghamton University

The Open Repository @ Binghamton (The ORB)

Mechanical Engineering Faculty Scholarship

Mechanical Engineering

7-2018

\title{
A parametric electrostatic resonator using repulsive force
}

Mark Pallay

Binghamton University--SUNY, mpallay1@binghamton.edu

Shahrzad Towfighian

Binghamton University--SUNY, stowfigh@binghamton.edu

Follow this and additional works at: https://orb.binghamton.edu/mechanical_fac

Part of the Mechanical Engineering Commons

\section{Recommended Citation}

Pallay, Mark and Towfighian, Shahrzad, "A parametric electrostatic resonator using repulsive force" (2018). Mechanical Engineering Faculty Scholarship. 18.

https://orb.binghamton.edu/mechanical_fac/18

This Article is brought to you for free and open access by the Mechanical Engineering at The Open Repository @ Binghamton (The ORB). It has been accepted for inclusion in Mechanical Engineering Faculty Scholarship by an authorized administrator of The Open Repository @ Binghamton (The ORB). For more information, please contact ORB@binghamton.edu. 


\title{
A parametric electrostatic resonator using repulsive force
}

\author{
Mark Pallay, Shahrzad Towfighian* \\ Binghamton University Mechanical Engineering Department, 4400 Vestal Parkway East, Binghamton, NY 13902, United States
}

\section{A R T I C L E I N F O}

\section{Article history:}

Received 31 October 2017

Received in revised form 21 February 2018

Accepted 1 April 2018

Available online 3 April 2018

\section{Keywords:}

MEMS

Parametric resonance

Repulsive-force

Electrostatic

Beams

\begin{abstract}
A B S T R A C T
In this paper, parametric excitation of a repulsive force electrostatic resonator is studied. A theoretical model is developed and validated by experimental data. A correspondence of the model to Mathieu's Equation is made to prove the existence and location of parametric resonance. The repulsive force creates a combined response that shows parametric and subharmonic resonance when driven at twice its natural frequency. The resonator can achieve large amplitudes of almost $24 \mu \mathrm{m}$ and can remain dynamically stable while tapping on the electrode. Because the pull-in instability is eliminated, the beam bounces off after impact instead of sticking to the electrode. This creates larger, stable trajectories that would not be possible with traditional electrostatic actuation. A large dynamic range is attractive for MEMS resonators that require a large signal-to-noise ratio.
\end{abstract}

(c) 2018 Elsevier B.V. All rights reserved.

\section{Introduction}

Vibrating micro-structures have played an important role in the development of micro-sensors because of their fast response time, low power consumption, and low bulk fabrication costs. MEMS (Microelectromechanical Systems) resonators are a class of MEMS devices that use the vibration of these structures for microphones [1,2], energy harvesters [3-7], accelerometers [8], signal filters [9-12], and many more applications [13]. Micro-sensors that require actuation, as opposed to those that rely on ambient vibration sources, typically use electrostatic forces for the ease of fabrication and power efficiency [13]. This usually comes at the cost of highly nonlinear behavior and the pull-in instability, which occurs when the attractive force between electrodes causes them to collapse. Nearly all electrostatic MEMS resonators have been designed around this usually undesirable phenomenon. Pull-in significantly limits the travel ranges of electrostatic MEMS sensors, which hinders performance. Because the sensitivity of capacitive sensors depend on the electrode voltage and travel range of the device, which are limited by pull-in, an electrostatic device that is not susceptible to pull-in would be very valuable [13].

In 2001, Lee and Cho [14] reported that two grounded electrodes would push away from each other if they were placed near a charged electrode on one side. This is not a pure repulsive force, but an attractive force that pulls the grounded electrodes apart. He

\footnotetext{
* Corresponding author.

E-mail addresses: mpallay1@binghamton.edu (M. Pallay), stowfigh@binghamton.edu (S. Towfighian).
}

and Ben Mrad used the same principle as Lee and Cho to create out-of-plane actuation [15-18] by flipping the design on its side. In this configuration the actuator is suspended above three electrodes (shown in Fig. 1). The actuator and center electrode are grounded while the side electrodes are charged. The resulting electrostatic field (visualized in Fig. 1b) pulls on the top of the beam more than the bottom because of the presence of the center electrode, which results in a net force away from the substrate. This is technically an attractive force; however it acts in the opposite direction of the electrodes and is referred to as repulsive to differentiate it from traditional electrostatic actuation. This design achieved large outof-plane actuation; however it requires a high voltage potential because of the weak forcing associated with fringe electrostatic fields. The primary focus of their study was for static actuation; however a large range of motion is very attractive for sensors.

The authors have recently extended the work by He and Ben Mrad to include dynamic applications [19-21]. It was shown that if the beam moves far enough from the electrodes, the force becomes attractive again. While the beam-electrode gap distance at which this occurs highly depends on the geometry, it can be as large as $60 \mu \mathrm{m}$, which provides a large repulsive regime. A large DC bias is required to push the static position of the beam far enough from the substrate to allow the beam to move without hitting the electrode. Because the beam and middle electrode are grounded, even if the beam strikes the electrode, it will not stick, but bounces off. This allows the device to operate when a collision occurs. We found softening nonlinearities associated with the repulsive force. Because the repulsive force uses the electrostatic fringe field, the system should also show parametric resonance, which can generate a large vibration amplitude. 


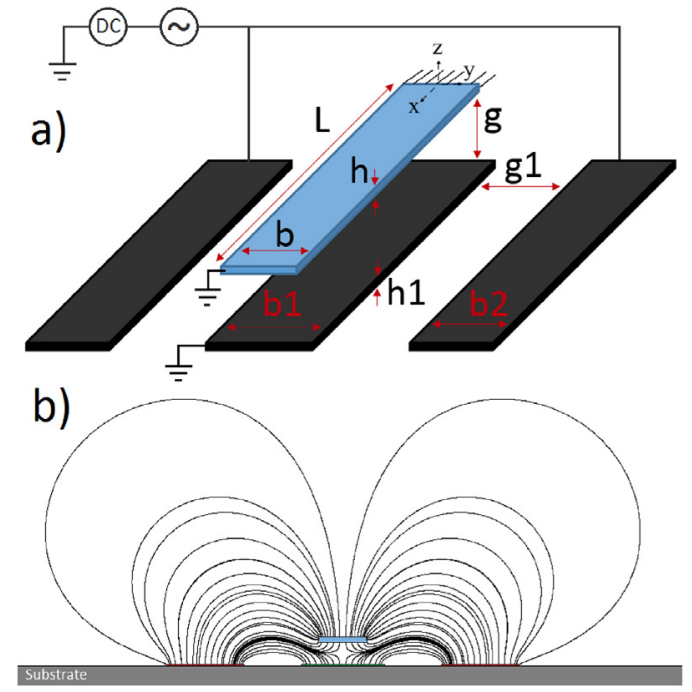

Fig. 1. (a) Electrode configuration of the resonator with cantilever boundary conditions. The actuator is shown in blue and the electrodes are black. A superimposed AC/DC voltage is applied to the side electrodes while the actuator and center electrode are grounded. (b) Electric field lines from an FEA simulation in COMSOL. The electric field generated from the side electrodes (red) pull on the top of the actuator (blue) more than bottom because of the presence of the center grounded electrode (green), resulting in a net force upwards. (For interpretation of the references to color in text/this figure legend, the reader is referred to the web version of the article.)

Parametric excitation (PE) involves exciting a system by changing the parameters of the system instead of applying a direct force in the direction of desired motion. Faraday discovered this phenomenon in the late 1800s and it has been applied to MEMS devices since the early 1990s [13,22-24]. PE can achieve very large oscillation amplitudes with relatively low levels of excitation. Because of this, many resonators have been designed to use PE rather than direct forcing [3,22,24-32]. In electrostatic MEMS, the electric field creates a distributed force on the surface of the beam, which is nonlinearly related to the gap distance. This can be seen as an electrical stiffness that acts in conjunction with the mechanical stiffness. By modulating the applied voltage, the overall stiffness of the beam can be controlled. This can drive the system into parametric resonance if the $\mathrm{AC}$ voltage is large enough to overcome non-conservative forces, such as viscous damping, and the driving frequency is inside an instability tongue (usually at twice the natural frequency) [13].

Parametric resonance (PR) can be practically achieved in MEMS by placing two electrodes along the length of a beam, which creates a negative stiffness that depends on the voltage level [31]. Because the beam is simultaneously pulled towards both electrodes, this design is susceptible to pull-in. Linzon et al. [24] designed a fringe field parametric resonator that eliminates the pull-in instability by placing the electrodes along the sides of the beam, instead of directly above and below. Instead of pulling towards the electrodes, the electrostatic force pulls the beam between the electrodes thus eliminating pull-in. However, this design requires an initial displacement or a curved micro-beam to operate because there is no net force on the resonator when the beam is at its rest position.

In this paper, parametric resonance of a repulsive force resonator with cantilever boundary conditions is studied (Fig. 1). The repulsive force allows for a large oscillation amplitude associated with PR without the risk of pull-in. Unlike [24], this electrode configuration generates a nonzero force on the actuator when it is at rest, so no initial displacement is necessary for its operation. This creates normal and parametric resonances. The nonlinearity of the electric field also creates subharmonic resonances of order two, which should occur at the primary parametric resonance. When a voltage is applied to the side electrodes, the electrostatic forces
Table 1

Beam geometry and material properties.

\begin{tabular}{lll}
\hline Parameter & Symbol & Value \\
\hline Cantilever length $(\mu \mathrm{m})$ & $L$ & 500 \\
Beam width $(\mu \mathrm{m})$ & $b$ & 17.5 \\
Beam height $(\mu \mathrm{m})$ & $h$ & 2 \\
Beam-electrode gap $(\mu \mathrm{m})$ & $g$ & 2 \\
Electrode gap $(\mu \mathrm{m})$ & $g 1$ & 20.5 \\
Electrode width $1(\mu \mathrm{m})$ & $b 1$ & 30 \\
Electrode width $2(\mu \mathrm{m})$ & $b 2$ & 28 \\
Electrode thickness $(\mu \mathrm{m})$ & $h 1$ & 0.5 \\
Elastic modulus $(\mathrm{GPa})$ & $\mathrm{E}$ & 165 \\
Density $\left(\mathrm{kg} / \mathrm{m}^{3}\right)$ & $\rho$ & 2330 \\
Poisson's ratio & $v$ & 0.22 \\
Force constant $\left(\mathrm{N} / \mathrm{m}^{6}\right)$ & $p_{5}$ & $-8.5695 \times 10^{14}$ \\
Force constant $\left(\mathrm{N} / \mathrm{m}^{5}\right)$ & $p_{4}$ & $1.7347 \times 10^{11}$ \\
Force constant $\left(\mathrm{N} / \mathrm{m}^{4}\right)$ & $p_{3}$ & $-1.2595 \times 10^{7}$ \\
Force constant $\left(\mathrm{N} / \mathrm{m}^{3}\right)$ & $p_{2}$ & $3.5574 \times 10^{2}$ \\
Force constant $\left(\mathrm{N} / \mathrm{m}^{2}\right)$ & $p_{1}$ & $-3.8677 \times 10^{-4}$ \\
Force constant $\left(\mathrm{N} / \mathrm{m}^{2}\right)$ & $p_{0}$ & $-1.1703 \times 10^{-7}$ \\
\hline
\end{tabular}

generate a positive stiffening effect on the cantilever, as confirmed in our previous experiment [19]. By changing the voltage on the side electrodes, the effective stiffness of the micro-structure can be changed. By modulating this at twice the natural frequency, the system should show parametric resonance. The motivation of this work is to study parametric resonance of this electrode configuration for sensors and filters where a large signal-to-noise ratio is required. For example, the proposed resonator can be used for a MEMS filter with a large dynamic range in signal processing applications [33].

The organization of this paper is as follows: In the next section, the formulation of the governing equation of motion is outlined. In Section 3, the relation to Mathieu's Equation is defined and the location of primary parametric resonance is estimated. Section 4 outlines the experimental methods. The model and experimental results are given in Section 5. Lastly, our conclusions based on the results are given in Section 6.

\section{Model derivation}

The actuator is modeled using Euler-Bernoulli beam theory. It is assumed to be linear-elastic polysilicon with dimensions given in Table 1. Axial displacement and other geometric nonlinearities are ignored. Despite the large oscillation, beam curvature is negligible because the length of the cantilever is more than an order of magnitude larger than the maximum deflection. The governing partial differential equation is given as,

$\rho A \frac{\partial^{2} \hat{w}}{\partial \hat{t}^{2}}+\hat{c} \frac{\partial \hat{w}}{\partial \hat{t}}+E I \frac{\partial^{4} \hat{w}}{\partial \hat{x}^{4}}+\hat{f}_{e}(\hat{w}) V^{2}=0$

where $\hat{w}$ is the $z$-direction beam displacement, $I$ is the moment of inertia, $V$ is the side electrode voltage, and $\hat{f}_{e}$ is the electrostatic force at a side electrode voltage of $1 \mathrm{~V}$. The electrostatic force profile is determined with a 2D COMSOL simulation and has been verified with our previous experiment [19]. Field effects at the tip are ignored because a 3D COMSOL simulation showed they made a negligible contribution to the electrostatic force. The force is assumed to be constant in the longitudinal direction. A 5th order polynomial is fit to the COMSOL data to be used with Eq. (1). The electrostatic force profile from COMSOL is shown in Fig. 2. 


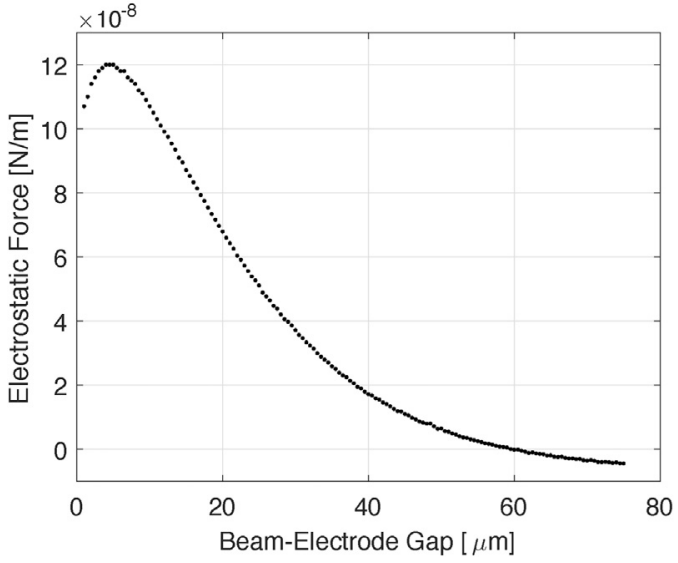

Fig. 2. Electrostatic force profile as determined through COMSOL.

Table 2

Nondimensional substitutions.

\begin{tabular}{ll}
\hline Parameter & Substitution \\
\hline$x$-Direction position & $x=\hat{x} / L$ \\
$z$-Direction position & $w=\hat{w} / h$ \\
Time & $t=\hat{t} / T$ \\
Damping & $c^{*}=\hat{c} L^{4} / E I T$ \\
Time constant $(s)$ & $T=\sqrt{\rho A L^{4} / E I}$ \\
Force constant $(m / N)$ & $r_{1}=L^{4} / E I h$ \\
\hline
\end{tabular}

Eq. (1) is nondimensionalized using the substitutions shown in Table 2, which yields the nondimensional equation of motion,

$\frac{\partial^{2} w}{\partial t^{2}}+c^{*} \frac{\partial w}{\partial t}+\frac{\partial^{4} w}{\partial x^{4}}+r_{1} V^{2} \overbrace{\sum_{j=0}^{5} p_{j} h^{j} w^{j}}^{f_{e}}=0$

where $p_{j}$ are constants from the 5 th order polynomial forcing fit. Eq. (2) is reduced into a set of coupled ordinary differential equations (ODE) through Galerkin's method. First, separation of variables is performed on Eq. (2), with the beam response approximated as,

$w(x, t) \approx \sum_{i=1}^{n} q_{i}(t) \phi_{i}(x)$

where $\phi_{i}(x)$ are the mode shapes of the beam, $q_{i}(t)$ are the time dependent generalized coordinates, and $n$ is the number of degrees of freedom (DOF). The mode shapes for the cantilever are given as,

$$
\begin{array}{r}
\phi_{i}(x)=\cosh \left(\alpha_{i} x\right)-\cos \left(\alpha_{i} x\right) \\
-\sigma_{i}\left(\sinh \left(\alpha_{i} x\right)-\sin \left(\alpha_{i} x\right)\right)
\end{array}
$$

where $\alpha_{i}$ are the square root of the nondimensional natural frequencies, and $\sigma_{i}$ are constants determined from the boundary conditions and mode to be considered. $\alpha_{i}$ and $\sigma_{i}$ are obtained from [34].

Once the mode shapes are known, Eq. (3) is plugged into the nondimensional equation of motion (Eq. (2)), which yields a coupled set of $n$ ODE's for $q_{i}$.

$$
\begin{aligned}
& \sum_{i=1}^{n} \phi_{i} \frac{\partial^{2} q_{i}}{\partial t^{2}}+c^{*} \sum_{i=1}^{n} \phi_{i} \frac{\partial q_{i}}{\partial t} \\
& \quad+\sum_{i=1}^{n} \frac{\partial^{4} \phi_{i}}{\partial x^{4}} q_{i}+r_{1} V^{2} \sum_{j=0}^{5} p_{j} h^{j}\left(\sum_{i=1}^{n} q_{i} \phi_{i}\right)^{j}=0
\end{aligned}
$$

Table 3

Air constants at 3.9 Torr.

\begin{tabular}{ll}
\hline Parameter & Value \\
\hline Quality factor & 130 \\
$k_{11}$ & 0.005 \\
$k_{12}$ & 4 \\
\hline
\end{tabular}

Because the mode shapes are orthogonal, the linear terms in Eq. (5) can be decoupled by multiplying through by $\phi_{k}$ and integrating over the length of the beam. This yields a set of $n$ ordinary differential equations,

$m_{i} \ddot{q}_{i}+c_{i} \dot{q}_{i}+k_{i} q_{i}+r_{1} V^{2} \sum_{j=0}^{5} \int_{0}^{1} f_{j k}\left(\sum_{i=1}^{n} \phi_{i} q_{i}\right)^{j} d x=0$

where nonlinear terms remain coupled and,

$m_{i}=\int_{0}^{1} \phi_{i}^{2} d x \quad k_{i}=\int_{0}^{1} \frac{d^{4} \phi_{i}}{d x^{4}} \phi_{i} d x \quad c_{i}=c^{*} m_{i}$

$f_{j k}=p_{j} h^{j} \phi_{k}$

For a one mode approximation, Eq. (6) becomes,

$m_{1} \ddot{q}_{1}+c_{1} \dot{q}_{1}+k_{1} q_{1}+r_{1} V^{2} \sum_{j=0}^{5} f_{j} q_{1}^{j}=0$

where

$f_{j}=p_{j} h^{j} \int_{0}^{1} \phi_{1}^{j+1} d x$

A linear damping model is used with Eq. (8). The damping coefficient is estimated using the relationship in Eq. (10) with the quality factor $(\mathrm{Q})$ and the first natural frequency $\left(\alpha_{1}^{2}\right)$.

$c=\frac{\alpha_{1}^{2}}{Q}$

The quality factor is given in Table 3 based on our previous study. Our study also verified the accuracy of the one mode model [19]. The driving voltage, $V$, is a sinusoidal AC voltage superimposed on a much larger DC voltage. A large DC voltage is required to give the beam enough room to vibrate without hitting the middle electrode.

Before Eq. (8) can be solved, air effects should be considered. In our experiment we found that the air spring effect dominates the nonlinear damping forces at low pressures. Therefore, linear damping and a nonlinear air spring term are used in Eq. (8). The linear damping coefficient is shown above in Eq. (10), and the air spring stiffness is given in Eq. (11).

$k_{\text {air }}=\frac{k_{11} \omega^{2}}{\left(q_{1}^{5}+1\right)} \frac{1}{1.0025+k_{12}\left(\omega^{2} / q_{1}^{4}+1\right)}$

The air spring term is necessary because of the large dynamic range of the device. As the beam gets close to the electrode, the air between the beam and substrate compresses causing the amplitude to saturate. This was seen in our previous experiment in [19] where the model, which did not account for air effects, overestimates the experimental results near the natural frequency. For the PE experiments in this study, the effect of air is even more pronounced because of the higher AC frequency and air pressure. Therefore, the air spring must be included in the model. The form of the air spring term is taken from [13], which is derived from the Reynolds equation. The constants are grouped into $k_{11}$ and $k_{12}$, which are determined through experiment. Values of $k_{11}$ and $k_{12}$ are given 
in Table 3. Eq. (11) is then added to Eq. (8), which yields the final equation of motion.

$m_{1} \ddot{q}_{1}+c_{1} \dot{q}_{1}+\left(k_{1}+k_{\text {air }}\right) q_{1}+r_{1} V^{2} \sum_{j=0}^{5} f_{j} q_{1}^{j}=0$

Once all terms are defined, Eq. (12) can be solved. The high nonlinearity associated with the electrostatic force and air spring create difficulties for more efficient computational methods such as shooting and harmonic balance. Therefore, Eq. (12) is solved with using long-time integration with MATLAB's ode45 solver.

\section{Mathieu's Equation}

To prove parametric resonance (PR) occurs in this system, Eq. (12) can be related to the damped Mathieu's Equation, shown below.

$\frac{d^{2} x}{d \tau^{2}}+c \frac{d x}{d \tau}+(\delta+\epsilon \cos (\tau)) x=0$

The linear Mathieu's Equation shows unbounded PR when $\delta=1 / 4,1,9 / 4$, etc., and $\epsilon$ is large enough to overcome the beam damping $c$ [35]. When there are certain nonlinearities in the system, such as the nonlinear stiffness from the electrostatic force, the response becomes bounded and does not grow indefinitely. Eq. (12) can be put in the form of Eq. (13) to find the driving frequency where PR will occur.

First, the electrostatic force is linearized about the static equilibrium point, $w=s$, using a Taylor Series because nonlinear terms have little influence on the location of PR.

$$
\begin{aligned}
& f_{e} \approx \beta_{1} q_{1}+\beta_{0}=\int_{0}^{1} \phi_{1}\left(\sum_{j=0}^{5} p_{j} h^{j} s^{j}+\right. \\
& \left.\sum_{j=1}^{5} j p_{j} h^{j} s^{j-1}\left(\phi_{1} q_{1}-s\right)\right) d x
\end{aligned}
$$

For a linearized force, the range of motion of the beam is small. Therefore, the air spring effect is negligible and will not have a major effect on the location of parametric resonance. Dropping $k_{\text {air }}$ and plugging the linearized force back into Eq. (12) yields,

$$
\begin{aligned}
& m_{1} \ddot{q}_{1}+c_{1} \dot{q}_{1}+k_{1} q_{1} \\
& \quad+r_{1}\left(V_{D C}+V_{A C} \cos (\omega t)\right)^{2}\left(\beta_{1} q_{1}+\beta_{0}\right)=0
\end{aligned}
$$

Substituting $\omega t=\tau$ and combining linear terms yields a nonhomogeneous form of Mathieu's Equation (more generally known as Hill's Equation) with two time scales.

$$
\begin{gathered}
\frac{d q_{1}^{2}}{d \tau^{2}}+2 \zeta \frac{\omega_{n}}{\omega} \frac{d q_{1}}{d \tau}+\left[\delta_{1}+\epsilon_{11} \cos (\tau)+\epsilon_{21} \cos (2 \tau)\right] q_{1} \\
=-\left(\frac{r_{1} \beta_{0} \tilde{V}_{D C}^{2}}{m_{1} \omega^{2}}+\epsilon_{10} \cos (\tau)+\epsilon_{20} \cos (2 \tau)\right)
\end{gathered}
$$

where

$$
\begin{aligned}
& 2 \zeta \omega_{n}=\frac{c_{1}}{m_{1}} \quad \omega_{n}=\sqrt{\frac{k_{1}}{m_{1}}} \\
& \delta_{1}=\frac{1}{\omega^{2}}\left(\omega_{n}^{2}+\frac{r_{1} \beta_{1} \tilde{V}_{D C}^{2}}{m_{1}}\right) \quad \epsilon_{1 i}=\frac{2 r_{1} \beta_{i} V_{D C} V_{A C}}{m_{1} \omega^{2}} \\
& \epsilon_{2 i}=\frac{r_{1} \beta_{i} V_{A C}^{2}}{2 m_{1} \omega^{2}} \quad \tilde{V}_{D C}^{2}=V_{D C}^{2}+\frac{V_{A C}^{2}}{2}
\end{aligned}
$$

Eq. (16) has parametric resonance arising from the linear term, and normal resonance associated with the forcing terms. As mentioned above, there are also two time scales for the $\epsilon_{1 i}$ and $\epsilon_{2 i}$ terms, $\tau$ and $2 \tau$. Because the DC voltage is typically much larger than the AC voltage, $\epsilon_{2 i}$ will be much smaller than $\epsilon_{1 i}$. Therefore, we focused our efforts on studying PR associated with the first term, though the effects of both are included in the analysis.

To find the primary parametric resonant frequency, the $\delta_{1}$ term is set equal to $1 / 4$ [35] and rearranged to find the AC voltage frequency, $\omega$,

$\omega=2 \sqrt{\left(\omega_{n}^{2}+\frac{r_{1} \beta_{1} \tilde{V}_{D C}^{2}}{m_{1}}\right)}$

where the square root term is the natural frequency with the stiffening effect of the applied voltage. Because $\beta_{1}$ is almost always positive, increasing DC voltage increases the natural frequency, which has been verified in our previous experiment [19]. From Eq. (18), one can see primary parametric resonance occurs at twice the natural frequency, as expected. The authors have previously mapped the primary instability tongue for a quality factor of 100 and $V_{D C}=187 \mathrm{~V}$ [20]. The threshold $A C$ voltage for parametric resonance was found to be approximately $7 V_{A C}$. At 3.35Torr and $V_{D C}=178 \mathrm{~V}$, the threshold voltage was measured at approximately $11.4 V_{A C}$.

It is also important to note that as the original equation of motion (Eq. (8)) has a quadratic non-linearity, the system will also show superharmonic and subharmonic resonances of order 2 and $1 / 2$ respectively. This means that at twice the natural frequency, there will be parametric and subharmonic resonance of order $1 / 2$. These can be differentiated from each other by observing the time signal and phase portrait.

\section{Experimental methods}

The resonators are fabricated in silicon using PolyMUMPs standard fabrication by MEMSCAP. A detailed outline of the process can be found in [36]. The beams have dimensions given in Table 1. A picture of a fabricated device is shown in Fig. 3. Dynamic tests are conducted using a Polytec MSA-500 laser vibrometer, which measures the beam velocity. The velocity signal is numerically integrated in the frequency domain to find dynamic displacement. Low frequency bins are set to zero to remove drift during integration.

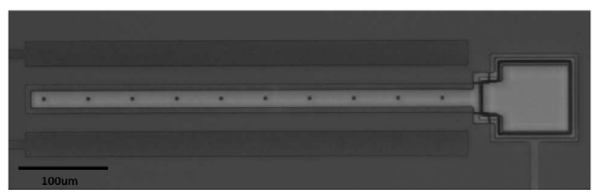

Fig. 3. PolyMUMPs fabricated resonator with anchor (right), beam (center), and side electrodes (top and bottom).

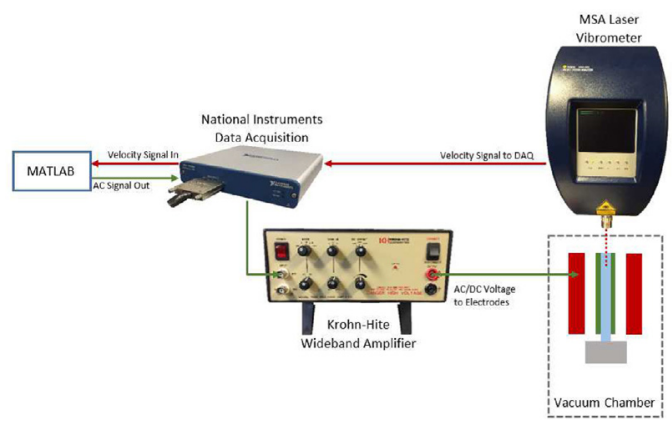

Fig. 4. Experimental setup with an NI Data Acquisition (interfaced with MATLAB), a Krohn-Hite Amplifier, a Vacuum Chamber, and a Micro Systems Analyzer. 


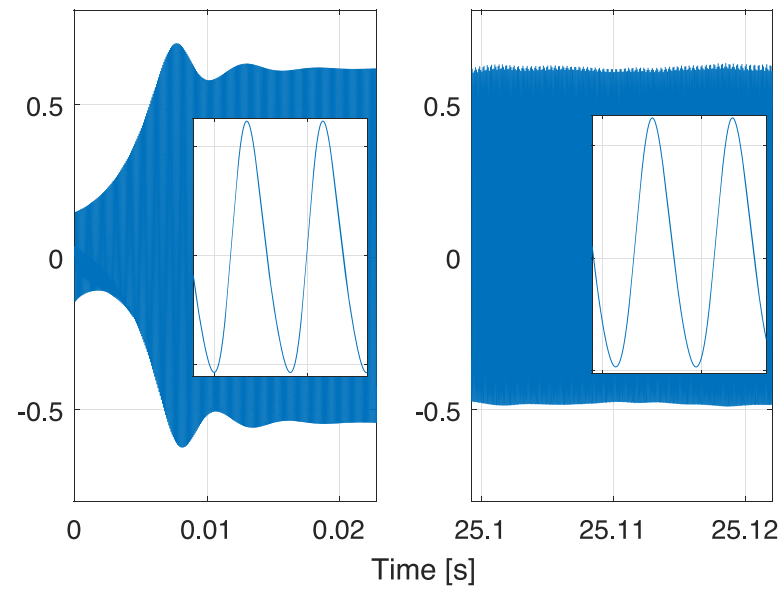

Fig. 5. Velocity time response for the model (left) and the experiment (right) at 16 $V_{A C}, 178 V_{D C}, 24,483 \mathrm{~Hz}$, and a pressure of 3.9Torr. Inlet shows zoomed in portion of steady state signal.

A schematic of the experimental setup is shown in Fig. 4. The beams are placed in a vacuum chamber and the pressure is reduced to approximately 3.5 Torr. The MATLAB Data Acquisition toolbox and a National Instruments USB 6366 Data Acquisition are used to apply voltages to the electrodes and to acquire data from the vibrometer. The AC output voltage is amplified, and a large DC offset is superimposed using a Krohn-Hite 7600 Wideband Power Amplifier. A Micro Systems Analyzer (MSA) Laser Vibrometer is used to measure the beam velocity. The natural frequency was previously measured to be approximately $12 \mathrm{kHz}$ at $178 V_{D C}$ [19]. Frequency sweeps around twice the natural frequency are performed. Beam velocity is measured at approximately $40 \mu \mathrm{m}$ from the tip.

\section{Results}

A comparison between the velocity time response of the model and the experiment at $16 V_{A C}, 178 V_{D C}$, and 3.9Torr is shown in Fig. 5. The beam motion is dominated by the first mode and agrees closely with the model. The experimental data is shifted upwards slightly, which is most likely a result of nonlinear damping. The model only accounts for the air spring effect with linear damping and thus the energy transferred to the squeezed air when the beam is close to the substrate is conserved. In the experiment however, there are nonlinear damping forces that increase significantly when the beam is close to the substrate. This takes energy out of the system and causes the beam to return to its nominal position with a slightly lower velocity than it left with.

To identify the frequency components, fast Fourier transformations (FFT) are performed on the time data from Fig. 5 and are shown in Fig. 6. The model predicts a multi-frequency response dominated by the driving frequency and its half, which is because of parametric resonance. The experimental results shows the two major frequency spikes as predicted by the model. The electrical noise is very small and does not contribute much to the overall signal. Therefore it is filtered out of the experimental data.

When the amplitude is small the beam shows subharmonic resonance. Fig. 7 shows the experimental time response when the frequency is set to $24,680 \mathrm{~Hz}$. Subharmonic resonance can be seen when a second peak appears in each oscillation cycle. This only occurs when the amplitude from parametric resonance is small. As the frequency is swept downward the parametric amplitude increases, such as in Fig. 5, and the subharmonic peak decreases until the response becomes sinusoidal.

The data in Fig. 5 correlate to a dynamic displacement that reaches upwards of $15 \mu \mathrm{m}$ peak to peak with a $2 \mu \mathrm{m}$ initial gap. At

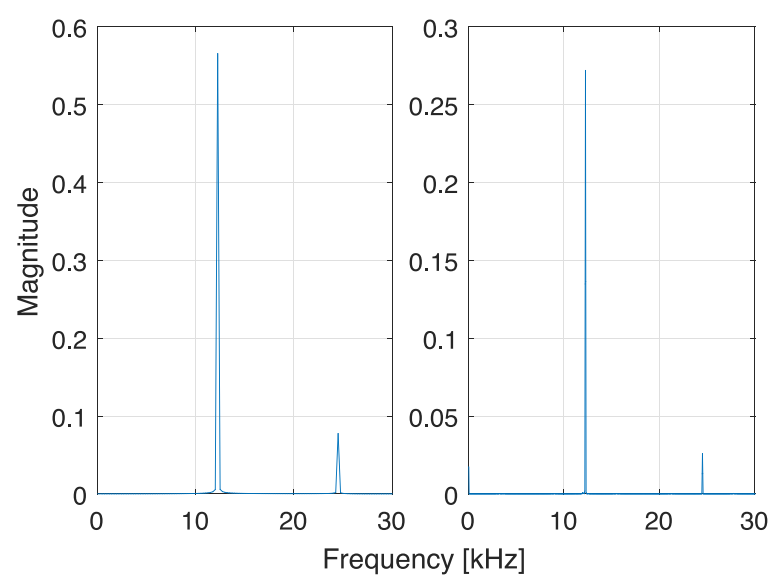

Fig. 6. Fast Fourier transform of the model data (left) and experimental data (right) at a driving frequency of $24,500 \mathrm{~Hz}$.

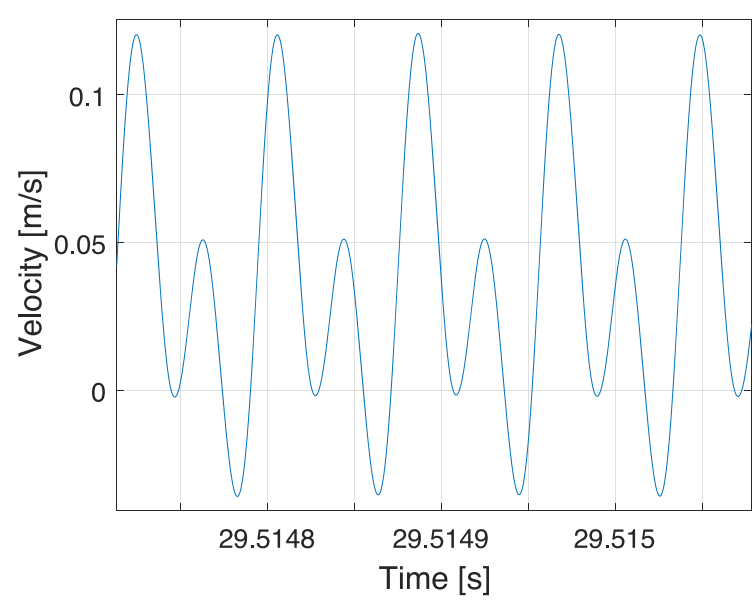

Fig. 7. Velocity time response at $24,680 \mathrm{~Hz}$ showing the appearance of subharmonic resonance.

other frequencies dynamic amplitudes as large as $24 \mu \mathrm{m}$ peak-topeak can be attained. This is possible because the DC voltage pushes the beam to a static position of over $10 \mu \mathrm{m}$, so the beam can oscillate a peak-to-peak of more than double that $(20 \mu \mathrm{m})$. This creates a very strong interaction with the surrounding air resulting from the large range of motion very close to the substrate. The authors have attempted to quantify this effect through a modified stiffness term that includes the air spring effect (Eq. (11)). This limits the amplitude as the beam approaches the substrate, which is observed in the experiment. Previous experiments from [19] show an overestimation of the model response at large amplitudes without taking the air spring effect into account. The effect of the air spring is more easily seen in the frequency response.

Next, frequency sweeps were conducted. First, a voltage just below the parametric threshold was tested to observe the pure subharmonic resonance, shown in Fig. 8. The window to see subharmonic resonance without parametric resonance is extremely small. At a pressure of approximately 3.4 Torr and $178 V_{D C}$, it is on the order of $10-15 \mathrm{mV}$, outside of which either shows no resonance peak or a high amplitude parametric response.

Fig. 8 shows the maximum peak amplitude while the driving frequency is swept around twice the natural frequency. The subharmonic resonance maintains a signal similar to Fig. 7, without ever becoming parametric. This can be seen more clearly in the phase portrait (Fig. 9), which shows the subharmonic phase portrait with a fold. 


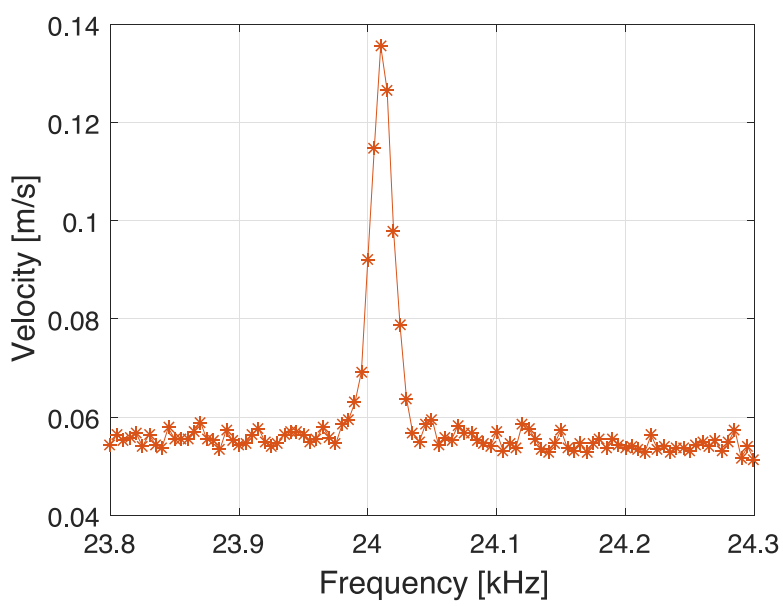

Fig. 8. Frequency response showing only subharmonic resonance at $178 V_{D C} 11.41$ $V_{A C}$, and 3.38 Torr. A separate beam was used for the subharmonic testing.

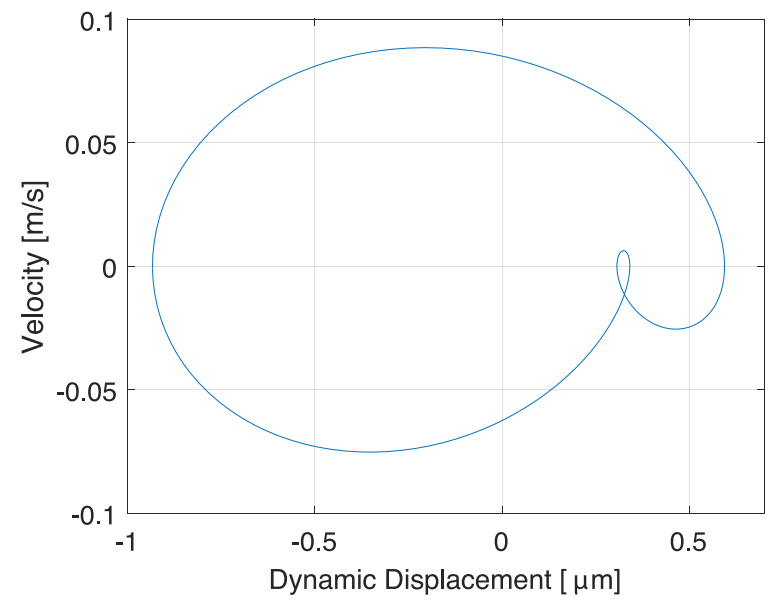

Fig. 9. Subharmonic phase portrait at $178 V_{D C} 11.41 V_{A C}, 24.01 \mathrm{kHz}$, and $3.38 \mathrm{Torr}$ showing the subharmonic loop.

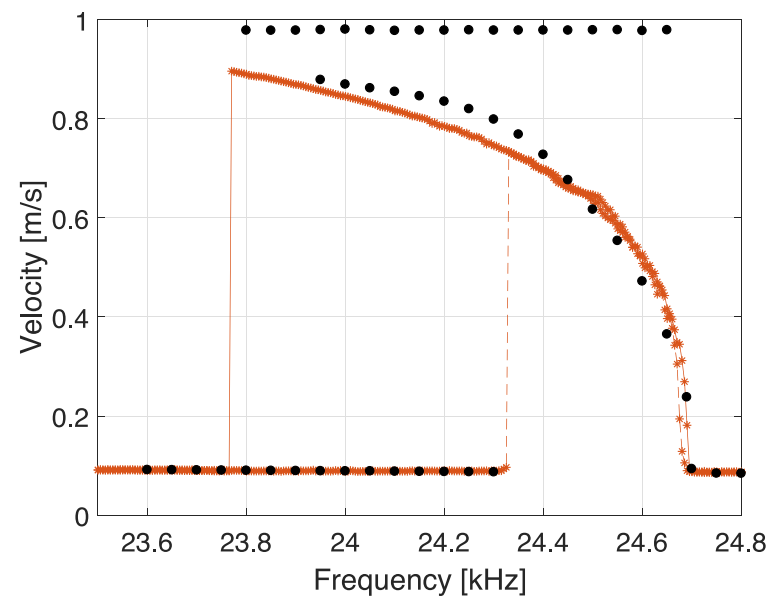

Fig. 10. Velocity frequency response at $16 V_{A C}, 178 V_{D C}$, and a pressure of 3.9 Torr (orange). The model (black) uses a quality factor of 130 with $k_{11}=0.005$ and $k_{12}=4$. (For interpretation of the references to color in text/this figure legend, the reader is referred to the web version of the article.)

Next, the voltage level was increased to excite parametric resonance. Velocity frequency responses for two voltage cases, along with the corresponding model results, are shown in Figs. 10 and 11.

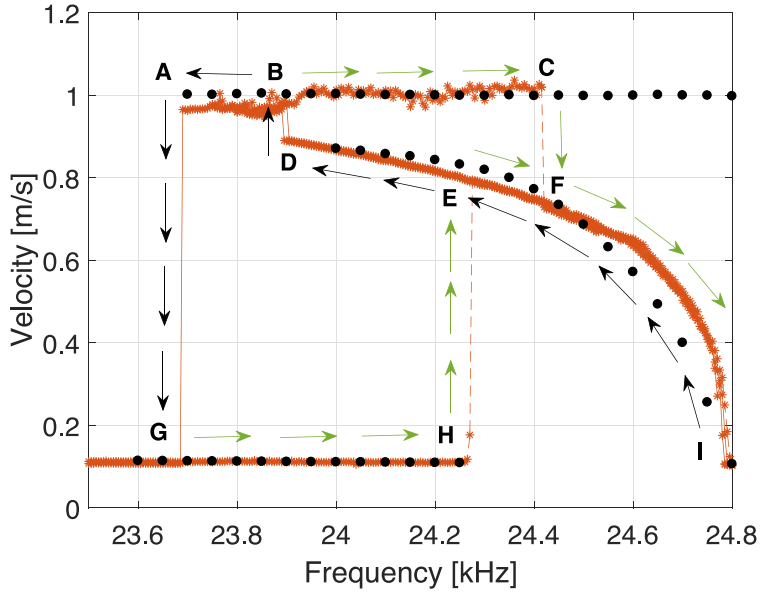

Fig. 11. Velocity frequency response at $20 V_{A C}, 178 V_{D C}$, and a pressure of 3.9Torr (orange). The model (black) uses a quality factor of 130 with $k_{11}=0.005$ and $k_{12}=4$. Points A, B, C, D, E, F, G, and $\mathrm{H}$ indicated where the beam experiences hysteresis. Black arrows indicate a downward frequency sweep while green arrows show an upward frequency sweep. (For interpretation of the references to color in text/this figure legend, the reader is referred to the web version of the article.)

The model shows very good agreement with the experiment. The system shows strong softening nonlinearities, which can be seen in the coefficients of the electrostatic force. There also exists a high amplitude hardening branch, which results from the air spring effect. This creates a frequency response that is highly nonlinear with up to three stable trajectories at a single frequency, all of which can be captured in the experiment if the AC voltage is large enough. The hardening branch is limited in the experiment because of the collision with the center electrode, which drops the response to the softening branch when enough energy is lost during the impact.

The resonator experiences hysteresis at multiple points on the frequency curve. Starting at I in Fig. 11, if the frequency is swept downward (black arrows) the response travels up the softening branch toward $\mathbf{D}$. At $\mathbf{D}$ the beam experiences hysteresis and jumps up to $\mathbf{B}$. If the frequency is swept downward from $\mathbf{B}$, it will go through hysteresis again at $\mathbf{A}$, jumping down to the lowest branch at G. If the frequency is swept upward (green arrows) from $\mathbf{B}$, it will experience hysteresis at $\mathbf{C}$, jumping back down to $\mathbf{F}$. For a forward sweep starting at $\mathbf{G}$, the beam shows hysteresis at $\mathbf{H}$ and jumps up to $\mathbf{E}$, and then travels back down the softening branch towards I.

Simulations show that for both voltage cases a very flat, high amplitude hardening branch exists. However, if the voltage is too small, such as for the case of $16 \mathrm{~V}$, the hardening amplitude branch will not be captured in the experiment. On the hardening branch, the beam is lightly tapping on the middle electrode, which also limits its response in addition to the squeezed air. The impacts can be seen in the velocity time response when higher order harmonics start to appear. A comparison between two time response curves, one with impacts, can be seen in Fig. 12.

The velocity data can be integrated in the frequency domain by taking a fast Fourier transform of the time data and dividing by the frequency. Figs. 13 and 14 , show the dynamic displacement plots for the velocity data in Figs. 9 and 10 respectively. The beam is traveling $24 \mu \mathrm{m}$ peak-to-peak at $20 V_{A C}$ on the hardening branch despite being anchored just $2 \mu \mathrm{m}$ from the substrate, which is made possible by the large DC voltage. The ability to travel almost $24 \mu \mathrm{m}$ and contact the middle electrode without pull-in is a unique characteristic that can be applied to create highly sensitive sensors. 


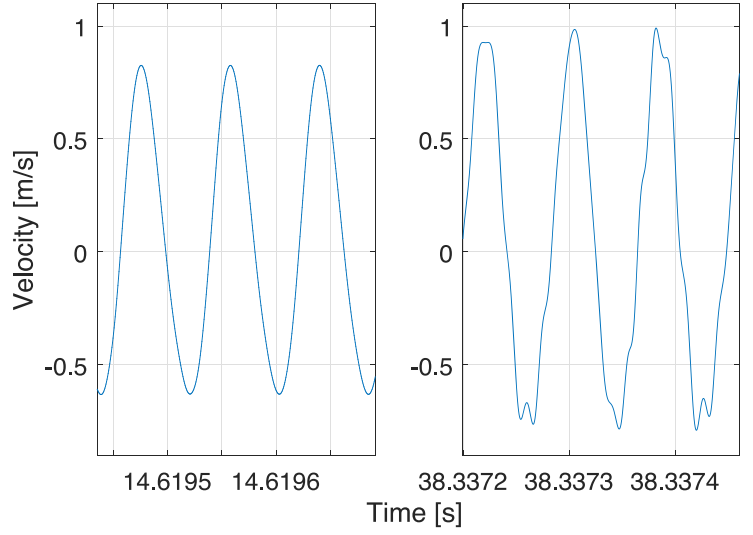

Fig. 12. Velocity time response of the data in Fig. 11 at $20 V_{A C}, 178 V_{D C}, 24,305 \mathrm{~Hz}$ (left), and $24,245 \mathrm{~Hz}$ (right). At $24,305 \mathrm{~Hz}$ on the lower branch no impact with the substrate occurs and the velocity signal is relatively sinusoidal. At $24,245 \mathrm{~Hz}$ on the higher branch, the beam is tapping on the electrode and exciting higher harmonics.

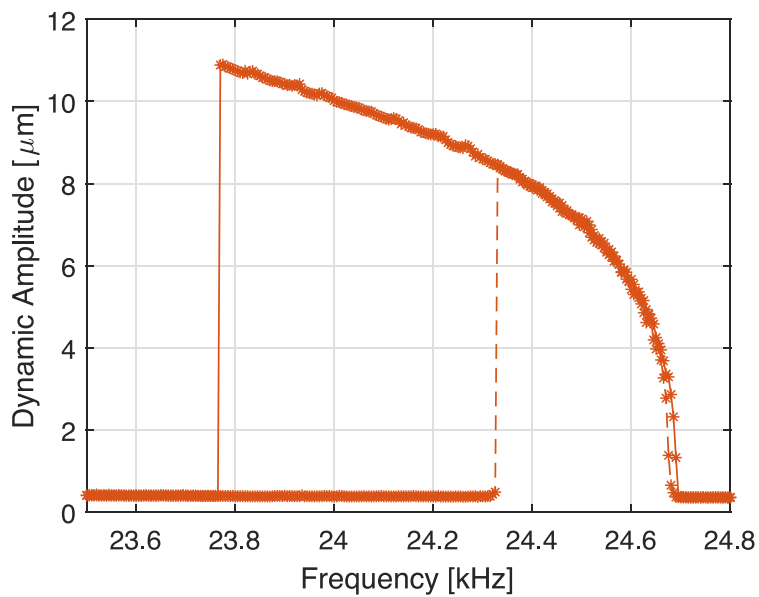

Fig. 13. Experimental dynamic displacement frequency response at $16 V_{A C}, 178 V_{D C}$, and a pressure of 3.9 Torr.

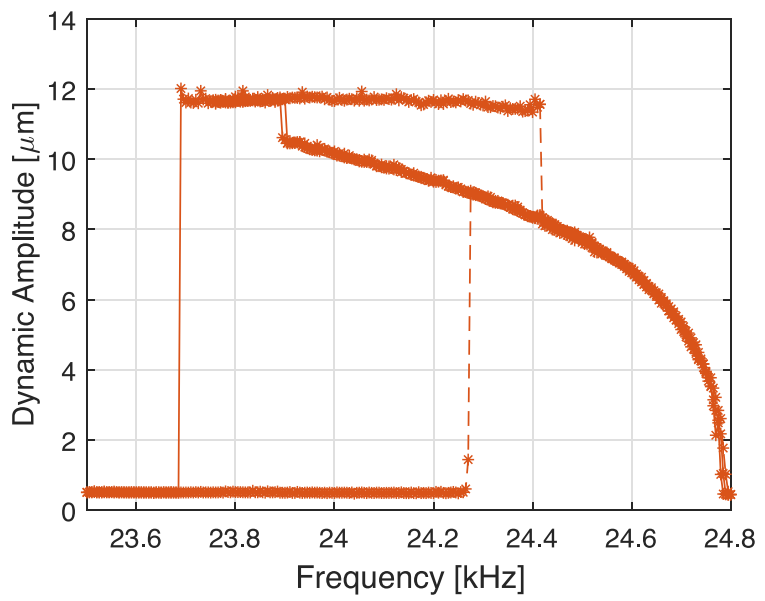

Fig. 14. Experimental dynamic displacement frequency response at $20 V_{A C}, 178 V_{D C}$, and a pressure of 3.9 Torr.

\section{Conclusions}

In this paper, parametric resonance of a repulsive force electrostatic actuator is examined. The repulsive force eliminates the pull-in instability and allows a large range of motion. A theoretical model of the resonator is developed and Mathieu's Equation is used to predict the location of the primary parametric resonance. Because of the nature of the repulsive force, parametric resonance and subharmonic resonance occur at twice the natural frequency. Large travel ranges of almost $24 \mu \mathrm{m}$ are observed and verified through experimental data. The repulsive force allows the beam to move in stable trajectories that tap on the center electrode without experiencing pull-in. Our results show the existence of three stable branches with one lightly tapping on the substrate, leading to high amplitudes as large as $24 \mu \mathrm{m}$ peak-to-peak that are physically attainable, unlike conventional electrostatic actuators. This repulsive force can be useful for sensors that require a large signal-to-noise ratio.

\section{Acknowledgments}

The authors would like to thank Ronald Miles from the Mechanical Engineering Department at Binghamton University for fruitful discussion regarding the experimental results. Authors are grateful for the financial support of this study by NSF ECCS grant \# 1608692.

\section{References}

[1] R.N. Miles, D. Robert, R.R. Hoy, Mechanically coupled ears for directional hearing in the parasitoid fly Ormia ochracea, J. Acoust. Soc. Am. 98 (6) (1995) 3059-3070.

[2] R.N. Miles, W. Cui, Q.T. Su, D. Homentcovschi, A MEMS low-noise sound pressure gradient microphone with capacitive sensing, J. Microelectromech. Syst. 24 (1) (2015) 241-248.

[3] Y. Jia, J. Yan, K. Soga, A.A. Seshia, Parametrically excited MEMS vibration energy harvesters with design approaches to overcome the initiation threshold amplitude, J. Micromech. Microeng. 23 (11) (2013) 114007.

[4] Y. Jia, A.A. Seshia, An auto-parametrically excited vibration energy harvester, Sens. Actuators: A. Phys. 220 (2014) 69-75.

[5] J.C. Park, J.Y. Park, Y.P. Lee, Modeling and characterization of piezoelectric d33-Mode MEMS energy harvester, J. Microelectromech. Syst. 19 (5) (2010) 1215-1222.

[6] U.M. Jamain, N.H. Ibrahim, R.A. Rahim, Performance analysis of zinc oxide piezoelectric MEMS energy harvester, IEEE International Conference on Semiconductor Electronics, Proceedings, ICSE (4) (2014) 263-266.

[7] S. Saadon, Y. Wahab, From Ambient Vibrations To Green Energy Source: MEMS Piezoelectric Energy Harvester for Low Frequency Application, 2015, pp. 59-63.

[8] M.I. Ibrahim, M.I. Younis, Enhancing the sensitivity of a resonant accelerometer, 2008 Proceedings of the ASME International Mechanical Engineering Congress and Exposition (2009) 241-247, 11.

[9] F. Huang, S. Fouladi, R. Mansour, A novel MEMS-based tunable dielectric resonator filter, IEEE MTT-S International Microwave Symposium Digest (2011) 2-5.

[10] B.K. Hammad, E.M. Abdel-Rahman, A.H. Nayfeh, Modeling and analysis of electrostatic MEMS filters, Nonlinear Dyn. 60 (3) (2010) 385-401.

[11] S. Shen, S. Young, Design and fabrication of a MEMS filter bank for hearing aids applications, 2nd Annual International IEEE-EMBS Special Topic Conference on Microtechnologies in Medicine and Biology. Proceedings (2002) 352-355.

[12] M. Ahmadi, G.A. Jullien, Programmable Mems, 2000, pp. 522-525.

[13] M.I. Younis, MEMS Linear and Nonlinear Statics and Dynamics, Springer, New York, 2011.

[14] K.B. Lee, Y.H. Cho, Laterally driven electrostatic repulsive-force microactuators using asymmetric field distribution, J. Microelectromech. Syst. 10 (1) (2001) 128-136.

[15] S. He, R. Ben Mrad, Large-stroke microelectrostatic actuators for vertical translation of micromirrors used in adaptive optics, IEEE Trans. Ind. Electron. 52 (4) (2005) 974-983.

[16] S. He, R. Ben Mrad, Design, modeling, and demonstration of a MEMS repulsive-force out-of-plane electrostatic micro actuator, J. Microelectromech. Syst. 17 (3) (2008) 532-547.

[17] S. He, R. Ben Mrad, Development of a multi-level repulsive force out-of-plane micro electrostatic actuator, IECON Proceedings (Industrial Electronics Conference), 2009, pp. 4020-4023.

[18] J. Chong, S. He, R. Ben Mrad, Control of a surface micromachined repulsive-force driven $2 \mathrm{D}$ micromirror, in: IEEE/ASME International Conference on Advanced Intelligent Mechatronics, AIM, 2010, pp. 1005-1007.

[19] M. Pallay, M. Daeichin, S. Towfighian, Dynamic behavior of an electrostatic MEMS resonator with repulsive actuation, Nonlinear Dyn. 89 (2) (2016) 1525-1538.

[20] M. Pallay, S. Towfighian, Parametric excitation of a repulsive force actuator; in: Proceedings of the ASME 2017 International Design Engineering Technical Conferences, August 6-9, Cleveland, OH, USA, DETC2017-67381, 2017, 7 p. 
[21] M. Ozdogan, M. Daeichin, A. Ramini, S. Towfighian, Parametric resonance of a repulsive force MEMS electrostatic mirror, Sens. Actuators A: Phys. 265 (2017) 20-31.

[22] D. Rugar, P. Grütter, Mechanical parametric amplification and thermomechanical noise squeezing, Phys. Rev. Lett. 67 (6) (1991) 699-702.

[23] K.L. Turner, S.A. Miller, P.G. Hartwell, N.C. MacDonald, S.H. Strogatz, S.G. Adams, Five parametric resonances in a microelectromechanical system, Nature 396 (6707) (1998) 149-152.

[24] Y. Linzon, B. Ilic, S. Lulinsky, S. Krylov, Efficient parametric excitation of silicon-on-insulator microcantilever beams by fringing electrostatic fields, J. Appl. Phys. 113 (16) (2013).

[25] J.F. Rhoads, S.W. Shaw, K.L. Turner, J. Moehlis, B.E. DeMartini, W. Zhang, Generalized parametric resonance in electrostatically actuated microelectromechanical oscillators, J. Sound Vib. 296 (4) (2006) 797-829.

[26] W.-M. Zhang, G. Meng, Nonlinear dynamic analysis of electrostatically actuated resonant MEMS sensors under parametric excitation, IEEE Sens. J. 7 (3) (2007) 370-380

[27] K.M. Harish, B.J. Gallacher, J.S. Burdess, J.A. Neasham, Experimental investigation of parametric and externally forced motion in resonant MEMS sensors, J. Micromech. Microeng. 19 (1) (2018).

[28] N. Kacem, S. Hentz, Bifurcation topology tuning of a mixed behavior in nonlinear micromechanical resonators, Appl. Phys. Lett. 95 (18) (2009) 2009-2012.

[29] Z. Hu, B.J. Gallacher, K.M. Harish, J.S. Burdess, An experimental study of high gain parametric amplification in MEMS, Sens. Actuators A: Phys. 162 (2) (2010) 145-154.

[30] M. Sharma, E.H. Sarraf, E. Cretu, Shaped combs and parametric amplification in inertial MEMS sensors, IEEE SENSORS 2013 - Proceedings 1 (1) (2013) 1-4

[31] S. Krylov, I. Harari, Y. Cohen, Stabilization of electrostatically actuated microstructures using parametric excitation, J. Micromech. Microeng. 15 (6) (2005) 1188-1204.
[32] D.I. Caruntu, I. Martinez, M.W. Knecht, Parametric resonance voltage response of electrostatically actuated Micro-Electro-Mechanical Systems cantilever resonators, J. Sound Vib. 362 (2016) 203-213.

[33] L. Lin, R.T. Howe, A.P. Pisano, Microelectromechanical filters for signal processing, J. Microelectromech. Syst. 7 (3) (1998) 286-294.

[34] S.S. Rao, Mechanical Vibrations, vol. 67, Pearson Prentice Hall, 2010

[35] A.H. Nayfeh, D.T. Mook, Nonlinear Oscillations, 1985.

[36] A. Cowen, B. Hardy, R. Mahadevan, S. Wilcenski, PolyMUMPs Design Handbook a MUMPs(r) process, 2011.

\section{Biographies}

Mark Pallay received his MS in Mechanical Engineering at Binghamton University in 2016. He is currently working towards his PhD at Binghamton where he works in the MEMS and Energy Harvesting Lab under Dr. Shahrzad Towfighian. His research focus is nonlinear dynamics in electrostatic MEMS for sensing applications.

Shahrzad Towfighian received her MS degree from Ryerson University in 2006 and her PhD degree in Mechanical Engineering from the University of Waterloo, Canada in 2011. She joined the Mechanical Engineering department at Binghamton University in Fall 2013. Her research interests include Micro-electro-mechanical sensors/actuators and energy harvesting. She develops mathematical modeling of electromechanical systems to study nonlinearities and their effect on the system performance. She discovers new ways of sensing and actuation for improving functionality of MEMS devices. 